\title{
Massive upper gastrointestinal bleeding from an accessory splenic artery mimicking isolated gastric varices
}

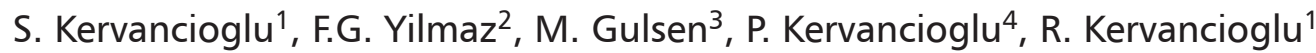 \\ ${ }^{1}$ Department of Radiology, Gaziantep University Medical Faculty, Gaziantep, Turkey \\ ${ }^{2}$ Department of Radiology, Islahiye State Hospital, Gaziantep, Turkey \\ ${ }^{3}$ Department of Gastroenterology, Gaziantep University Medical Faculty, Gaziantep, Turkey \\ ${ }^{4}$ Department of Anatomy, Gaziantep University Medical Faculty, Gaziantep, Turkey
}

[Received 25 April 2013; Accepted 29 May 2013]

\begin{abstract}
Knowledge of anatomical variations of coeliac trunk and its branches is important for surgeons and interventional radiologists planning surgical and radiological interventions. We describe a case of a 42-year-old male with an accessory splenic artery (ASA) originating from the left gastric artery ( $L G A)$ running in the wall of gastric fundus and mimicking isolated gastric varices, which was observed during endoscopy. Bleeding from this artery was massive and was managed with endovascular coil embolisation. Coeliac angiography of the patient with upper gastrointestinal bleeding showed that the coeliac trunk divided into 3 arteries: the LGA, the splenic artery, and the right hepatic artery. Additionally, the variations of ASA and the left hepatic artery arising from LGA, and the left and right inferior phrenic arteries arising from ASA were identified. This case is the first to be presented in the literature with ASA originating from LGA that was situated in the gastric wall where inferior phrenic arteries arose from the ASA. (Folia Morphol 2013; 72, 4: 366-370)
\end{abstract}

Key words: accessory splenic artery, upper gastrointestinal bleeding, coeliac trunk, variation

\section{INTRODUCTION}

Upper gastrointestinal bleeding (UGIB) is one of the most common causes of gastrointestinal emergency. It refers to bleeding in the gastrointestinal tract anywhere from the proximal oesophagus to the duodenum above the ligament of Treitz. The causes of UGIB are classified into variceal and non-variceal in origin. Common causes of UGIB include varices, peptic ulcer, benign and malignant tumours, ischaemia, gastritis, arteriovenous malformations such as Dieulafoy lesions, Mallory-Weiss tears, trauma and iatrogenic causes $[2,3]$. For an effective treatment of UGIB, timely and accurate diagnosis (location and aetiology) is needed. Over the past 2 decades, trans- catheter arterial embolisation has been performed for the management of haemorrhage for a variety of indications, and interventional radiology has been shown to be effective at controlling haemorrhage and decreasing mortality [3]. Knowledge of variations of coeliac trunk and its branches would enable surgeons and interventional radiologists to conserve these vessels and aid in the prevention of malpractice.

The splenic artery (SA) is the largest branch of the coeliac trunk and remarkable for its tortuosity. From its origin, it runs inferiorly for a short distance, then turns left and runs horizontally behind the stomach, along the upper border of the pancreas. Near the tail of the pancreas, it enters in the lienorenal ligament 
and then divides into terminal branches that enter the hilum of the spleen [14]. Many earlier reports showed that SA has well-known variations in the tortuosity, course, position and number of the branches; however, there is no report about an accessory splenic artery (ASA) lying in the gastric wall $[4-8,11]$. We describe a patient with massive gastric haemorrhage caused by the ASA that mimics isolated fundal gastric varices. This case is the first to be presented in the literature with ASA originating from left gastric artery (LGA) that was situated in the posterior fundal gastric wall, left hepatic artery (LHA) originated from LGA, and left and right inferior phrenic arteries (IPAs) originating from ASA.

\section{CASE REPORT}

A 42-year-old male was admitted to our hospital with haematemesis and melaena. He had no recent history of steroidal or non-steroidal anti-inflammatory drug use or alcohol consumption. On admission, his blood pressure was $90 / 65 \mathrm{~mm} \mathrm{Hg}$. The laboratory data at admission were as follows: haemoglobin $8.1 \mathrm{~g} / \mathrm{dL}$, haematocrit $25 \%$, platelets $165,000 / \mathrm{mm}^{3}$, blood urea nitrogen $46 \mathrm{mg} / \mathrm{dL}$, creatinine $0.54 \mathrm{mg} / \mathrm{dL}$, total protein $4.26 \mathrm{~g} / \mathrm{dL}$, total bilirubin $1.14 \mathrm{mg} / \mathrm{dL}$, aspartate aminotransferase $16 \mathrm{IU} / \mathrm{L}$, alanine aminotransferase $11 \mathrm{IU} / \mathrm{L}$, alkaline phosphatase $45 \mathrm{IU} / \mathrm{L}$, gamma-glutamyltranspeptitase $7 \mathrm{IU} / \mathrm{L}$, prothrombin time $13 \mathrm{~s}$, INR 1.1. The hepatitis B and hepatitis C viral markers were negative. Upper gastrointestinal endoscopy revealed that the fundus was covered with blood clots. Transfusion of $3 \mathrm{U}$ of packed red blood cells ( $p$-RBCs) was provided, and intravenous administration of proton pump inhibitor was initiated. The next day melaena was repeated and haemoglobin was $7.4 \mathrm{~g} / \mathrm{dL}$. The second endoscopy revealed type 1 isolated gastric varices with dilated and tortuous vessels at fundus, without an active haemorrhage. The patient was transfused with 2 units of $\mathrm{p}$-RBCs. On the following days, abdominal ultrasonography (USG) and computed tomography (CT) were normal. Colour Doppler USG revealed normal portal and splenic veins that excluded the portal hypertension and the splenic vein thrombosis. Computed tomography angiography (CTA) was performed to understand the vascular anatomy of the stomach. It revealed an abnormal artery originated from the LGA (Fig. 1). This abnormal artery was seen in the muscular and submucosal layers of the stomach and reached to upper part of the spleen and supplied this region

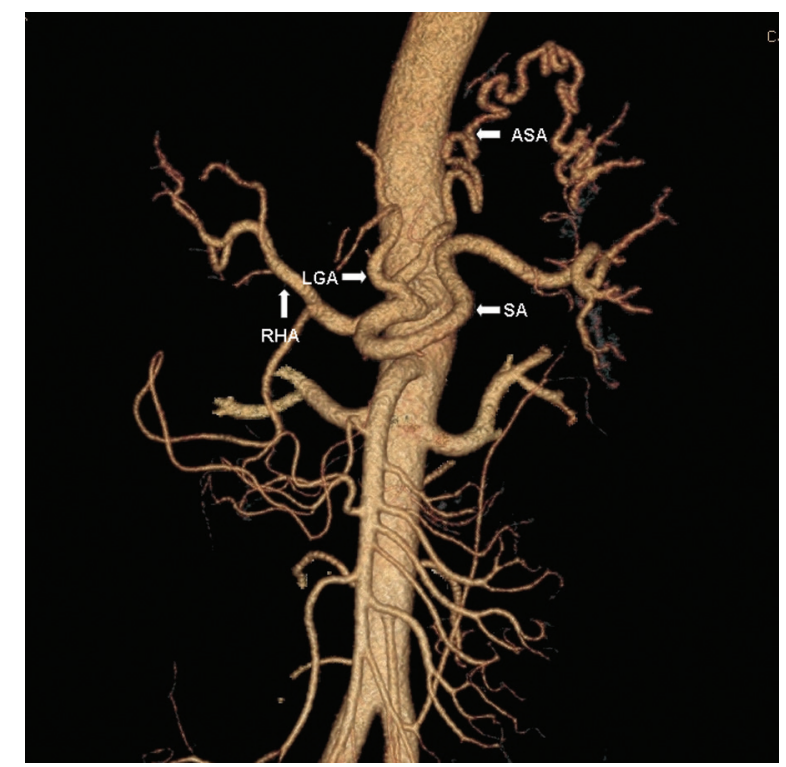

Figure 1. Computed tomography angiography shows coeliac trunk and its branches. ASA - accessory splenic artery; LGA — left gastric artery; RHA — right hepatic artery; SA — splenic artery.

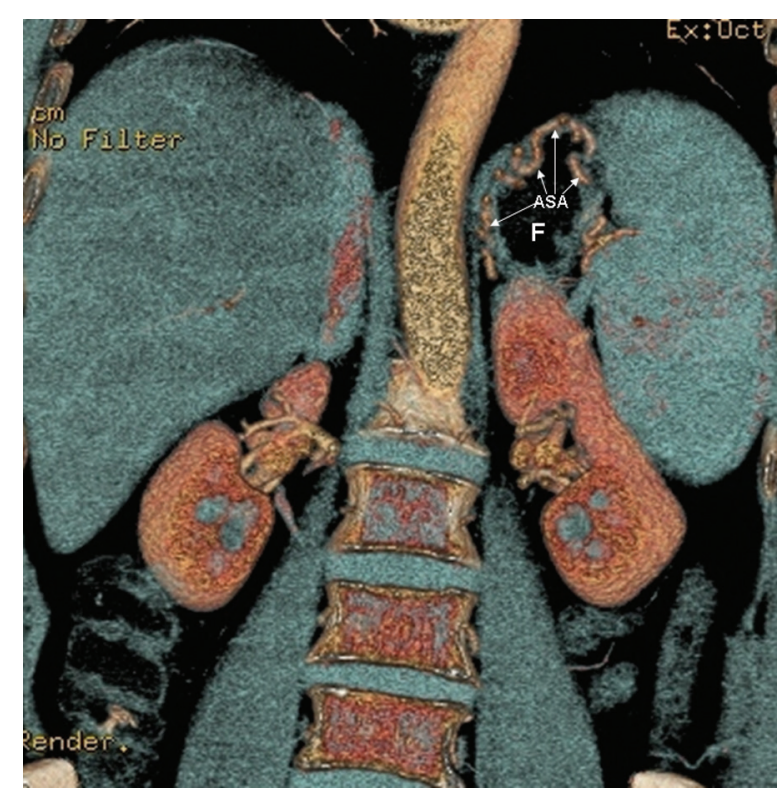

Figure 2. Computed tomography angiography shows accessory splenic artery (ASA) that is running in the gastric wall of the fundus (F).

(Fig. 2). Digital subtraction angiography (DSA) was performed to confirm the diagnosis and treat the cause of bleeding. Coeliac angiography showed that the coeliac trunk divided into 3 arteries: LGA, SA, and right hepatic artery (Fig. 3). LHA was originated from LGA (Fig. 4). An abnormal tortuous artery also originated from LGA and supplied the upper part of the spleen (Fig. 5). Right and left IPAs originated from 


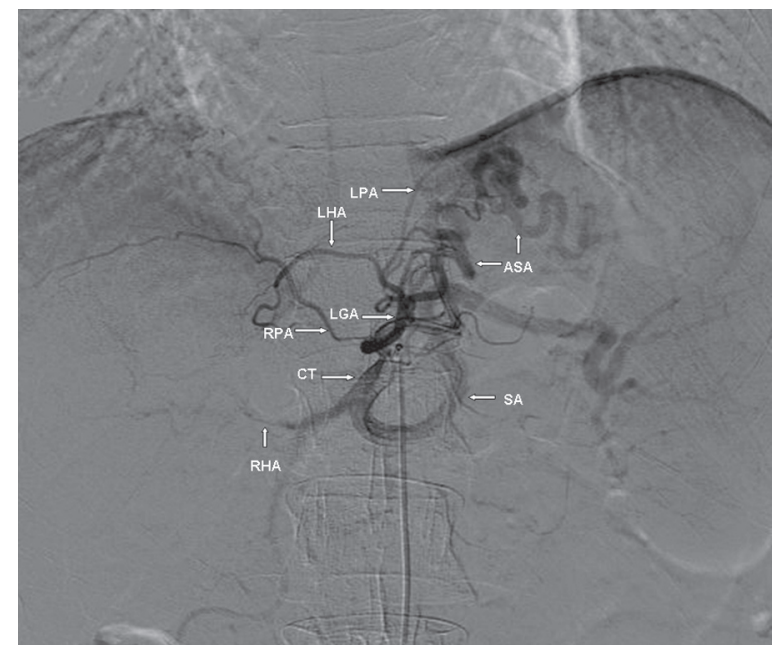

Figure 3. Coeliac angiography shows the branches of coeliac trunk (CT) with left gastric artery (LGA), splenic artery (SA), and right hepatic artery (RHA). Left hepatic artery (LHA) and accessory splenic artery (ASA) originate from LGA; right phrenic artery (RPA) and left phrenic artery (LPA) originate from ASA.

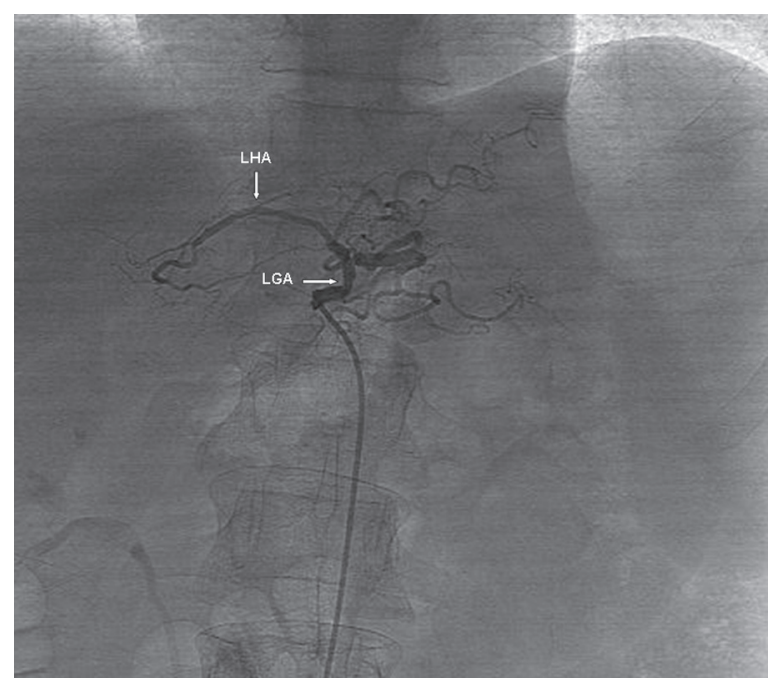

Figure 4. Angiography of left gastric artery (LGA) distal to origin of accessory splenic artery shows the normal LGA and variation of left hepatic artery (LHA).

this abnormal artery (Figs. 3, 6). As there was a normal $\mathrm{SA}$, this abnormal artery seen at CT angiography and DSA was the second SA, so we named it as ASA. Due to the tortuosity, we could not reach to distal part of the ASA with microcatheter, thus it was embolised with 8 hilal embolisation microcoils (Cook, DK) in the proximal part of the artery (Fig. 6). Although no splenic infarction occurred, mild abdominal pain was seen after the transcatheter embolisation. The patient has never experienced UGIB for the last 18 months.

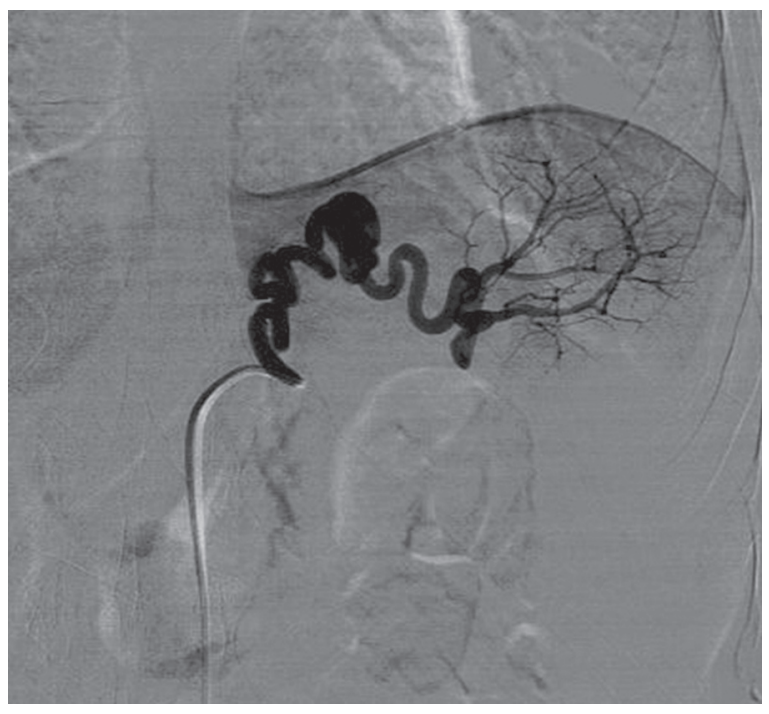

Figure 5. Digital subtraction angiography of accessory splenic artery shows the tortuosity of the artery which is feeding the upper part of the spleen.

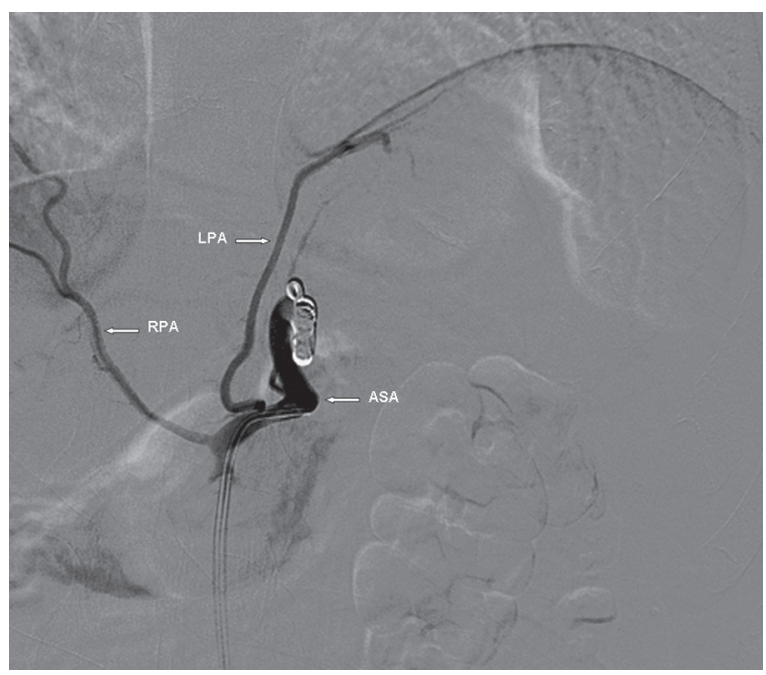

Figure 6. After coil embolisation of the accessory splenic artery (ASA), only right phrenic artery (RPA) and left phrenic artery (LPA) are seen.

\section{DISCUSSION}

The causes of UGIB are classified into variceal and non-variceal in origin. Variceal rupture is one of the important causes of UGIB, and it is a major cause of death and complications in patients with liver cirrhosis [2].

Gastric varices are usually presented with massive bleeding and have increased patient mortality [2]. Sarin et al. [9] classified gastric varices as gastro-oesophageal varices and isolated gastric varices (IGVs). 
Gastro-oesophageal varices consist of extension of oesophageal varices to the fundus and are categorised as type 1 and 2. Type 1 is more common and extends along the lesser curvature. Type 2 extends along the fundus, and this tends to be longer and more tortuous than type 1. IGV occur in the absence of oesophageal varices and are categorised into 2 types. Type 1 is located in the fundus and tends to be tortuous and complex. Type 2 is located in the body, antrum, or around the pylorus. In the presented case, because of the tortuosity and the location of ASA, endoscopy revealed fundal varicose vascularity, and the initial diagnosis was type 1 IGV. However, abdominal CT and colour Doppler USG excluded portal hypertension and splenic vein thrombosis. CTA and DSA revealed that this fundal tortuous vascularity was an artery which lies in the muscular and submucosal layers of the posterior wall of the fundus and then reach the upper part of the spleen to supply this region. As this artery is the main artery of the upper part of the spleen, it was thought that this was ASA originated from LGA. Thus the cause of UGIB in this case was not variceal, as it was an arterial bleeding.

CTA revealed an abnormal artery originated from LGA (Fig. 1). This abnormal artery was seen in the muscular and submucosal layers of stomach and reached to upper part of the spleen (Fig. 2). DSA was performed to confirm the diagnosis and treat the cause of bleeding.

In the present case, angiography also showed 2 more variations. One of them is LHA originated from LGA, and the other are left and right IPAs originated from ASA. Usually, LHA arises from the common hepatic artery (CHA). According to Bergman et al. [1], the aberrant origin of LHA from LGA is reported with variable frequency ranging from $10 \%$ to $19 \%$. It is important to know the hepatic artery variations in liver transplantation [4]. Inferior phrenic arteries usually arise from aorta, but occasionally they arise from coeliac trunk, a common aortic origin with coeliac trunk, renal artery or LGA. To the best of our knowledge, such a variation of IPAs has not been reported in the literature.

Normally, coeliac trunk is the first anterior branch of abdominal aorta, and divides into LGA, CHA and SA [14]. In the present case, the coeliac trunk divided into LGA, SA and right hepatic artery. The SA is the largest branch of coeliac trunk, but sometimes has an aberrant origin, and arises from aorta, CHA, LGA, or superior mesenteric artery $[4,7,10,12]$. In the present case, there was a normal SA regarding the origin and course.

At the end of the $4^{\text {th }}$ week of intrauterine life, a number of paired primitive vessels in the form of vitelline arteries supply the yolk sac. In the later weeks, these arteries gradually fuse to form coeliac trunk, superior mesenteric and inferior mesenteric arteries in the dorsal mesentery of the gut, which are present in adult life $[7,15]$. The anatomical variations of the coeliac trunk observed in the present study may result from the persistence of normally disappearing branch/branches or incomplete fusion or malfusion among these primitive arteries. Congenital absence, total duplication, intrahepatic course, aberrant origins from the aorta, superior mesenteric or LGA, and variations of terminal branching pattern of SA have been reported in the literature $[4,5,7,8,11$, 13]. Padmalatha et al. [6] reported an ASA originated from left gastroepiploic artery in a cadaver. The ASA of Padmalatha et al. [6] rose from left gastroepiploic artery which was already a branch of SA. The ASA in the present case is different, as it rose from LGA. Our case is the first in the literature in terms of ASA running in the gastric wall and mimicking type 1 IGV with additional variations of LHA originated from LGA, and IPAs originated from ASA.

Surgical treatment of such patients is complicated, and splenectomy and partial gastrectomy have been proposed as traditional methods of treatment. We preferred endovascular management with embolisation of the ASA. In this context, we intended to occlude the ASA at both the entrance and exit of the gastric wall. Due to the tortuosity, we could not reach to distal part of the ASA with a microcatheter, and it was embolised only in the proximal part of the artery. In the following 18 months, the patient did not have an experience of gastric bleeding, and the endoscopy revealed the disappearance of the fundal varicose vascularity.

\section{CONCLUSIONS}

In conclusion, this case with ASA and LHA originating in the LGA, and left and right IPAs originating from the ASA is the first presented case in the literature. Knowledge of variation in the branches of coeliac trunk is important for the surgeons operating in the area of supracolic region, and for radiologists dealing with interventional radiology. Such variation of ASA should be kept in mind in the absence 
of portal hypertension in terms of laboratory tests and radiological findings, when type 1 IGV is seen in upper gastrointestinal endoscopy. Bleeding from this abnormal artery can be life-threatening, but is managed easily with endovascular coil embolisation.

\section{REFERENCES}

1. Bergman RA, Afifi AK, Miyauchi R (2006) Illustrated encyclopedia of human anatomic variation. URL:http://www. anatomyatlases.org/Anatomic Variants/Anatomy HP.shtml.

2. Classen M, Tytgat G, Lightdale C (2010) Gastroenterological Endoscopy. Thieme, New York.

3. Loffroy RF, Abualsaud BA, Lin MD, Rao PP (2011) Recent advances in endovascular techniques for management of acute nonvariceal upper gastrointestinal bleeding. World J Gastrointest Surg, 3: 89-100.

4. Oran I, Yesildag A, Memis A (2001) Aortic origin of right hepatic artery and superior mesenteric origin of splenic artery: two rare variations demonstrated angiographically. Surg Radiol Anat, 23: 349-352.

5. Ozan H, Onderoglu S (1997) Intrapancreatic course of the splenic artery with combined pancreatic anomalies. Surg Radiol Anat, 19: 409-411.

6. Padmalatha K, Ramesh BR, Prakash BS, Balachandra N Mamatha $Y$ (2010) Accessory splenic artery from left gastroepiploic artery. Int J Anat Var, 3: 106-107.
7. Pandey SK, Bhattacharya S, Mishra RN, Shukla VK (2004) Anatomical variations of the splenic artery and its clinical implications. Clin Anat, 17: 497-502.

8. Sahni AD, Jit BI, Gupta CNM, Gupta DM, Harjeet E (2003) Branches of the splenic artery and splenic arterial segments. Clin Anat, 16: 371-377.

9. Sarin SK, Lahoti D, Saxena SP, Murthy NS, Makwana UK (1992) Prevalence, classification and natural history of gastric varices: a long-term follow-up study in 568 portal hypertension patients. Hepatology, 16: 1343-1349.

10. Slaba S, Karam R, Ingea H, Haddad S, Noun R, Aoun N, Atallah N (1998) Aberrant origin of the splenic artery. J Radiol, 79: 434-435.

11. Slaba S, Sfeir S, Nassar J, Noun R, Checrallah A, Tamraz J (2005) Unusual variation of the splenic artery. J Radiol, 86: 657-658.

12. Sponza M, Pozzi MR, Pozzi MF (1993) Arterial anatomy of the celiac trunk and the superior mesenteric artery with computerized tomography. Radiol Med, 86: 260-267.

13. Spring DW (1994) Congenital absence of the splenic artery. Cardiovasc Intervent Radiol, 7: 303-305.

14. Standring S ed. (2008) Gray's anatomy: the anatomical basis of clinical practice. $39^{\text {th }} \mathrm{Ed}$. Churchill Livingstone, New York.

15. Venieratos D, Panagouli E, Lolis E, Tsaraklis A, Skandalakis $P$ (2012) A morphometric study of the celiac trunk and review of the literature. Clin Anat, doi: 10.1002/ca.22136. [Epub ahead of print]. 